\title{
A Complete Analytic Inversion of Supernova Lines in the Sobolev Approximation
}

\author{
Daniel Kasen ${ }^{1,2}$ \\ dnkasen@panisse.lbl.gov \\ David Branch ${ }^{2,1}$ \\ branch@mail.nhn.ou .edu \\ E. $\operatorname{Baron}^{2,1}$ \\ baron@mail.nhn.ou.edu \\ and \\ David Jeffery ${ }^{3}$ \\ jeffery@kestrel.nmt.edu
}

\begin{abstract}
We show that the shape of P-Cygni line profiles of photospheric phase supernova can be analytically inverted to extract both the optical depth and source function of the line - i.e. all the physical content of the model for the case when the Sobolev approximation is valid. Under various simplifying assumptions, we derive formulae that give $S(r)$ and $\tau(r)$ in terms of derivatives of the line flux with respect to wavelength. The transition region between the minimum and maximum of the line profile turns out to give especially interesting information on the optical depth near the photosphere. The formulae give insights into the relationship between line shape and physical quantities that may be useful in interpreting observed spectra and detailed numerical calculations.
\end{abstract}

Subject headings: line: formation - line: profiles — line: identification — radiative transfer — supernovae

\footnotetext{
${ }^{1}$ Lawrence Berkeley National Laboratory, Berkeley, CA 94720

${ }^{2}$ Department of Physics and Astronomy, University of Oklahoma, Norman, OK 73019

${ }^{3}$ Department of Physics, New Mexico Tech, Socorro, NM 87801
} 


\section{Introduction}

The Sobolev approximation (Sobolev 1960; Castor 1970; Rybicki \& Hummer 1978) allows for a simplified solution to the radiative transfer equation in media with high velocity gradients, such as supernovae. The Sobolev approximation has been used to calculate synthetic spectra in stars with strong winds (Castor \& Nussbaumer 1972; Pauldrach et al. 1986) and to fit observed supernova spectra and place constraints on explosion models (Branch et al. 1983; Jeffery \& Branch 1990; Mazzali et al. 1992; Deng et al. 2000). Typically these models assume spherical symmetry and ignore continuous opacity. Despite the simplifying assumptions, the synthetic spectra fit the data quite well.

To calculate line profiles in the Sobolev case two physical quantities must be specified: (1) $\tau(r)$ : the optical depth of a line as a function of radius (often assumed to be a power law), and (2) $S(r)$ : the source function of the line as a function of radius (often assumed to be a resonant scattering source function). The Sobolev approximation has most often been used, like more sophisticated radiative transfer techniques, in a direct analysis of data, where the physical quantities are specified or calculated and the resulting spectrum is compared to observed data. On the other hand, there has been some interest in taking the inverse approach, i.e. using the line shape of observed data to infer the physical conditions in the atmosphere. Fransson \& Chevalier (1989) showed that the emissivity could be calculated for forbidden lines by differentiating the observed line profile with respect to wavelength and estimated the effects of electron scattering. Ignace \& Hendry (2000), using the Sobolev approximation, derived an analytic formula that gave a combination of $S(r)$ and $\tau(r)$ as a function of the derivative of the red side of an emission feature of arbitrary optical depth. The run of the optical depth of a line is then given if one specifies a form for the source function. For instance $S(r)$ in the case of pure resonance scattering is given by:

$$
S(r)=I_{\mathrm{ph}} W(r)
$$

where

$$
W(r)=\frac{1}{2}\left(1-\sqrt{1-\left(\frac{r_{\mathrm{ph}}}{r}\right)^{2}}\right)
$$

is the dilution factor (Mihalas 1978). $I_{\mathrm{ph}}$ is the intensity from the photosphere and $r_{\mathrm{ph}}$ the radius of the photosphere.

However, in supernova atmospheres the source function may deviate strongly from pure resonance scattering. In fact the failure of Sobolev models to properly fit the shape of some spectral lines - in particular net emission features - is basically because the source function is usually assumed that of pure resonant scattering, not because of direct limitations in the Sobolev approximation itself. The source function is an interesting quantity in its own right 
and ideally an inversion would extract both $S(r)$ and $\tau(r)$ from the line profile. In what follows we show how the degeneracy found by Ignace \& Hendry (2000) can be broken for photospheric phase supernovae and derive analytic expressions for both $\tau(r)$ and $S(r)$ using the shape of the entire line profile, thus providing a complete solution to the inverse problem.

We derive the formulae assuming spherical symmetry, an expanding line-scattering atmosphere surrounding a sharp continuum-emitting photosphere that absorbs any flux scattered back onto it, no continuous opacity, and no line blending. Even when these assumptions are not strictly valid the formulae should still give considerable insight into the physical conditions in the atmosphere. On the other hand, the limitations of the formulae provide an interesting result in their own right - they clearly show what type of features are impossible under the above assumptions, making it is obvious where more complicated scenarios must be invoked to explain a spectrum.

\section{The Sobolev Approximation}

In the most often used Sobolev model, one begins with a perfectly sharp, spherical photosphere that emits radiation as a blackbody, and which is surrounded by a moving atmosphere with large velocity gradient. The basic idea behind the Sobolev approximation is that a photon emitted from the photosphere only interacts with a line in the small region of the atmosphere where the photon is Doppler shifted into resonance with the line. Since the source function can be assumed to be constant over this small resonance region, the solution of the radiative transfer equation is greatly simplified. It also becomes simpler to visualize line formation, as the line flux at a given wavelength comes from a 2-dimensional resonance surface in the atmosphere. The criterion for the validity of the Sobolev approximation is that the resonance regions be sufficiently small, and this is characterized by the ratio of the atmosphere's thermal velocity (or mean microturbulent velocity, if significant) to the velocity scale height (i.e the velocity range over which temperature, density, and occupation numbers change by a factor of order 2 (Jeffery 1993)). Quantitative accuracy is found for velocity ratios $\lesssim 0.1$ (Olson 1982). For photospheric phase supernovae thermal velocities are of order $10 \mathrm{~km} \mathrm{~s}^{-1}$ and velocity scale heights are $\sim 10^{3} \mathrm{~km} \mathrm{~s}^{-1}$, giving a ratio of $\sim 10^{-2}$.

For supernova atmospheres, homology is established shortly after the explosion, so that $v=r / t$, where $t$ is the time since explosion. In this case the resonance surface for a wavelength is a plane perpendicular to the line of sight. We label the line of sight with a coordinate $\mathrm{z}$, with origin at the center of expansion and with $z$ increasing away from the observer (see Figure 1). A plane at coordinate $z$ has a z-component velocity of $v_{z}=z / t=$

$z v_{\mathrm{ph}} / r_{\mathrm{ph}}$ where $v_{\mathrm{ph}}$ is the velocity of the photosphere. This plane is then responsible for the 
line flux at a wavelength $\lambda=\lambda_{0}\left[1+\left(z / r_{\mathrm{ph}}\right)\left(v_{\mathrm{ph}} / c\right)\right]$, i.e the rest wavelength, $\lambda_{0}$, Doppler shifted by the z-component velocity.

The flux at a given wavelength is calculated by integrating over all the characteristic rays of the corresponding plane. Figure 1 shows that the formation of the line profile breaks up schematically into three regions. For $z \geq 0$, the flux is redshifted with respect to the line center so we call this the red side. This leads to an expression for the flux as an integral over impact parameter $\mathrm{p}$ (the coordinate perpendicular to the line of sight):

$$
\begin{gathered}
\frac{F(z)}{2 \pi}=\int_{0}^{r_{\mathrm{ph}}} I_{\mathrm{ph}} p d p+\int_{r_{\mathrm{ph}}}^{\infty} S(r)(1-\zeta(r)) p d p \\
=\frac{1}{2} r_{\mathrm{ph}}^{2} I_{\mathrm{ph}}+\int_{r_{\mathrm{ph}}}^{\infty} S(r)(1-\zeta(r)) p d p
\end{gathered}
$$

where $\zeta(r)=e^{-\tau(r)}$ and $F(z)$ is the observed flux (apart from a factor of $1 / D^{2}$, where $D$ is the distance to the supernova) at wavelength $\Delta \lambda=\lambda-\lambda_{0}=\lambda_{0} z v_{\mathrm{ph}} / c r_{\mathrm{ph}}=\lambda_{0} z / c t$. The first term in Eqn. (3) accounts for the flux coming directly from the photosphere, and the second term for photons scattered or created to emerge along the line of site. We have assumed for convenience an infinite atmosphere although none of our results is altered in the case that the atmosphere terminates at some radius $r_{\max }$.

For $z<0$, the integral has three terms in general, with the third term in Eqn. (4) now representing the region where material intervening between the photosphere and the observer leads to absorption of the continuum radiation:

$$
\begin{aligned}
\frac{F(z)}{2 \pi} & =\int_{0}^{p_{0}} I_{\mathrm{ph}} p d p+\int_{p_{0}}^{\infty} S(r)(1-\zeta(r)) p d p+\int_{p_{0}}^{r_{\mathrm{ph}}} I_{\mathrm{ph}} \zeta(r) p d p \\
& =\frac{1}{2} p_{0}^{2} I_{\mathrm{ph}}+\int_{p_{0}}^{\infty} S(r)(1-\zeta(r)) p d p+\int_{p_{0}}^{r_{\mathrm{ph}}} I_{\mathrm{ph}} \zeta(r) p d p .
\end{aligned}
$$

The limit $p_{0}$ is given by the $p$ location of the spherical photosphere for a given $z$, namely

$$
p_{0}= \begin{cases}\sqrt{r_{\mathrm{ph}}^{2}-z^{2}} & \text { for }-r_{\mathrm{ph}}<z<0 \\ 0 & \text { for } z \leq-r_{\mathrm{ph}}\end{cases}
$$

and the first term of Eqn. (4) is identically zero for $z \leq-r_{\mathrm{ph}}$. We call the part of the line profile where $z<-r_{\mathrm{ph}}$ the blue side and the part where $-r_{\mathrm{ph}}<z<0$ the mid region. 


\section{The Inversion Formulae}

\subsection{Inversion for $\zeta(r)$ for $r_{\mathrm{ph}}<r<\sqrt{2} r_{\mathrm{ph}}$}

We consider the inversion of each region of the line in turn, beginning with the mid region. The mid region of the line profile turns out to be only sensitive to the optical depth of the line near the photosphere. Using Eqn. (4), we change the integration variable from $p$ to $r=\sqrt{p^{2}+z^{2}}$, and divide through by $I_{\mathrm{ph}}$ :

$$
\frac{r_{\mathrm{ph}}^{2}}{2} f(z)=\int_{|z|}^{r_{\mathrm{ph}}} r d r+\int_{r_{\mathrm{ph}}}^{\infty} s(r)(1-\zeta(r)) r d r+\int_{r_{\mathrm{ph}}}^{\sqrt{z^{2}+r_{\mathrm{ph}}^{2}}} \zeta(r) r d r,
$$

where we have defined $s(r)=S(r) / I_{\mathrm{ph}}$ and $f(z)=F(z) /\left(\pi I_{\mathrm{ph}} r_{\mathrm{ph}}^{2}\right)$ (i.e. the total flux divided by the continuum flux). $I_{\mathrm{ph}}$ has been assumed to be constant over the line profile.

Written this way we see that the term involving the source function is independent of $z$ and so contributes a constant amount to the flux for every point in the mid region. The derivative of the mid region is therefore independent of the source function. The change in flux from a velocity surface at $z$ to one at $z-\Delta z$ is due only to the fact that a bit more of the photosphere is now obscured by the optical depth of the line. One then expects the derivative $\frac{d f}{d z}$ to depend only on the optical depth.

Since the terms in Eqn. (5) only depend on $z$ in the limits of the integral we can differentiate the integrals using Leibnitz' rule:

$$
\frac{d}{d z} \int_{\xi(z)}^{\eta(z)} g(t) d t=g(\eta) \frac{d \eta}{d z}-g(\xi) \frac{d \xi}{d z}
$$

Applying Eqn. (6) to Eqn. (5) allows us to solve for $\zeta(r)$ :

$$
\zeta\left(r=\sqrt{r_{\mathrm{ph}}^{2}+z^{2}}\right)=1-\frac{r_{\mathrm{ph}}^{2}}{2|z|} \frac{d f}{d z}=1-\frac{\lambda_{0}^{2}}{2|\Delta \lambda|} \frac{d f}{d \Delta \lambda}\left(\frac{v_{\mathrm{ph}}}{c}\right)^{2}
$$

which is valid for $-r_{\mathrm{ph}}<z<0$. In using Eqn. (7) to calculate $\zeta(r)$ from a spectrum, one can choose either $\Delta \lambda, z$, or $r$ as the independent parameter. For instance, from $\Delta \lambda$ (which is always less than zero for Eqn. [7]) the other two parameters are determined by

$z=r_{\mathrm{ph}}\left(\Delta \lambda / \lambda_{0}\right)\left(c / v_{\mathrm{ph}}\right)$ and $r=\sqrt{r_{\mathrm{ph}}^{2}+z^{2}}$. The photospheric radius is itself given by $r_{\mathrm{ph}}=v_{\mathrm{ph}} t$; however if the time since explosion is not known, one can still determine $\zeta$ as a function of the scaled distance $r / r_{\mathrm{ph}}$.

Eqn. (7) gives us some immediate insight into the relationship between line shape and optical depth. The steepness of the mid region (once the photospheric velocity has been 
scaled out) is a direct indication of the Sobolev optical depth. If no line feature exists, then $\frac{d f}{d z}=0$ and hence $\zeta=1$ (i.e $\tau=0$ ). Thus the absence of a feature implies either negligible line optical depth or the breakdown of our assumptions - in this formalism there is no choice for the source function that allows a line to "erase" itself. A stair-step mid region could be a signal that the optical depth near the photosphere is oscillating between small and large values (i.e. the medium is clumpy).

Note that since $\zeta \leq 1$, Eqn. (7) implies that the derivative $\frac{d f}{d z}$ is always greater than or equal to zero; i.e. the mid region always increases (or is flat) to the red. The appearance of a rising hump in the mid region, could indicate that the stated assumptions do not hold.

The fact that $\frac{d f}{d z} \geq 0$ also implies that an emission feature cannot peak blueward of its rest wavelength (at the most it can remain flat into the mid region as one would have for a detached atmosphere). However, the peaks of emission features are indeed found to be blue-shifted, both in real data and in spherically symmetric NLTE models. Jeffery \& Branch (1990) and Duschinger et al. (1995) attribute the blueshift to an NLTE effect where a large source function near the photosphere enhances the flux in the mid region. Under our assumptions this cannot be correct, since Eqn. (5) shows that the shape of the mid region is independent of the source function. Various second order physical effects, not included in the inversion formulae, could possibly explain the blueshifts, for example: continuous opacity added to the model could preferentially extinguish photons from the red side of the envelope; an absorption from another line to the red could cut into the emission peak; a large slope in the continuum could shift the peak; clumpiness of the photosphere (Wang \& Hu 1994) could break the spherical symmetry of the problem; relativistic effects can cause a significant blueshift for high photospheric velocities ( $\geq$ approximately $15,000 \mathrm{~km} / \mathrm{s}$ (Jeffery 1993)); or line-scattered light could be diffusely reflected off and blueshifted by the photosphere, causing a blueshift of the emission peak (Chugai 1988).

A few points must be made concerning the applicability of Eqn.(7): (1) near the rest wavelength the equation may not yield reliable results, since the $\Delta \lambda$ in the denominator goes to zero and must be delicately canceled by the flux derivative also going to zero. Thus any noise in the flux derivative (which must be evaluated numerically from the data) will be inflated at small $\Delta \lambda$. (2) one can not extract realistic values for $\tau \gg 1$ since $\zeta$ depends exponentially on $\tau$. One will know $\tau$ is large but not its exact value; (3) at late times (the nebular phase) the photosphere may become negligibly small and so there is no mid region - in this case, as we will see, our analysis reduces to that of Ignace \& Hendry (2000); (4) Eqn. (7) only gives the value of $\zeta$ for the radial region $r_{\mathrm{ph}}<r<\sqrt{2} r_{\mathrm{ph}}$. This is expected to be the region of highest density opacity in the atmosphere and so our formula for $\zeta(r)$ is interesting in itself. For example, if an atmosphere's density scales like $r^{-8}$ (a density law 
often used in spectral analysis; e.g. Millard et al. (1999)) then at $r=\sqrt{2} r_{\text {ph }}$ the optical depth has already fallen to $1 / 16$ of its photospheric value. Nevertheless, in the following we show how it is possible to extend the solution for $\zeta(r)$ to arbitrary $r$ by using information from the blue and red sides of the line profile.

\subsection{Inversion for $S(r)$}

We next consider the inversion of the red side of the line, which will allow us to solve for the source function. Changing variables in Eqn. (3), we see that the flux is now given by a source term plus an unobstructed photosphere term:

$$
\frac{r_{\mathrm{ph}}^{2}}{2} f(z)=\frac{1}{2} r_{\mathrm{ph}}^{2}+\int_{\sqrt{r_{\mathrm{ph}}^{2}+z^{2}}}^{\infty} s(r)(1-\zeta(r)) r d r .
$$

The second term in Eqn. (3) is a constant with respect to $z$ since the photosphere is always completely unobscured for $z>0$. The same technique of differentiating the integral allows us to solve for $s(r)$ :

$$
\begin{aligned}
s\left(r=\sqrt{r_{\mathrm{ph}}^{2}+z^{2}}\right) & =-\frac{r_{\mathrm{ph}}^{2}}{1-\zeta(r)} \frac{1}{2 z} \frac{d f}{d z} \\
& =-\frac{1}{1-\zeta(r)} \frac{\lambda_{0}^{2}}{2 \Delta \lambda} \frac{d f}{d \Delta \lambda}\left(\frac{v_{\mathrm{ph}}}{c}\right)^{2}
\end{aligned}
$$

which is valid for all $z \geq 0$ and the independent parameter can be chosen to be any of $\Delta \lambda, z$, or $r$. This is essentially the same result derived by Ignace \& Hendry (2000). Because Eqn. (7) together with Eqn. (13) (see below) gives $\zeta$ everywhere, Eqn. (9) can be used to determine the source function at all radii above the photosphere. Note if $\tau=0$ then $\zeta=1$ and Eqn. (9) is undefined - if a line has no optical depth it is of course impossible to determine its source function. For large optical depth, $\zeta=0$, and the shape of the red side depends on the source function only. Since $s \geq 0$ and $\zeta \leq 1$ we must have $\frac{d f}{d z} \geq 0$ on the red side - the red side always decreases (or stays flat) to the red. One cannot have humps or even a redshifted emission peak. Redshifts can occur due to non-Sobolev radiative transfer effects, but these are likely to be very small for supernovae (Hamann 1981). 


\subsection{Inversion for $\zeta(r)$ for $r>\sqrt{2} r_{\mathrm{ph}}$}

Finally the flux from the blue side of the profile will allow us to extend the solution of $\zeta$ to large $r$. The flux is given by a source term plus a fully obstructed photosphere:

$$
\frac{r_{\mathrm{ph}}^{2}}{2} f(z)=\int_{|z|}^{\infty} s(r)(1-\zeta(r)) r d r+\int_{|z|}^{\sqrt{r_{\mathrm{ph}}^{2}+z^{2}}} \zeta(r) r d r
$$

The same differentiation technique yields:

$$
\zeta\left(r=\sqrt{r_{\mathrm{ph}}^{2}+z^{2}}\right)=\zeta(|z|)+s(|z|)\{1-\zeta(|z|)\}-\frac{r_{\mathrm{ph}}^{2}}{2|z|} \frac{d f}{d z}
$$

which is valid for $z<-r_{\mathrm{ph}}$. Making use of spherical symmetry, Eqn. (9) can be used to replace the second term in Eqn. (11) with

$$
s(|z|)(1-\zeta(|z|))=-\frac{r_{\mathrm{ph}}^{2}}{2 z_{+}} \frac{d f\left(z_{+}\right)}{d z}
$$

where $z_{+}=\sqrt{z^{2}-r_{\mathrm{ph}}^{2}}$. Combining Eqns. (11) and (12) we obtain:

$$
\begin{aligned}
& \zeta\left(r=\sqrt{z^{2}+r_{\mathrm{ph}}^{2}}\right)=\zeta(r=|z|) \\
& -\frac{\lambda_{0}^{2}}{2}\left(\frac{v_{\mathrm{ph}}}{c}\right)^{2}\left\{\left[\frac{1}{\Delta \lambda} \frac{d f}{d \Delta \lambda}\right]_{\Delta \lambda=\frac{\lambda_{0}}{c t} \sqrt{z^{2}-r_{\mathrm{ph}}^{2}}}+\left[\frac{1}{|\Delta \lambda|} \frac{d f}{d \Delta \lambda}\right]_{\Delta \lambda=-\frac{|z| \lambda_{0}}{c t}}\right\} .
\end{aligned}
$$

where $|z|>r_{\mathrm{ph}}$ is the independent parameter for evaluating $\zeta\left(r=\sqrt{z^{2}+r_{\mathrm{ph}}}\right)$ from $\zeta(r=|z|)$ and $\frac{d f}{d z}$. Given $\zeta(r)$ for $r \in\left[n r_{\mathrm{ph}}, \sqrt{n+1} r_{\mathrm{ph}}\right]$, Eqn. (13) allows us to evaluate $\zeta(r)$ for $r \in$ $\left[\sqrt{n+1} r_{\mathrm{ph}}, \sqrt{n+2} r_{\mathrm{ph}}\right]$ where $n \geq 1$ is an integer. Beginning with $\zeta(r)$ for $r \in\left[r_{\mathrm{ph}}, \sqrt{2} r_{\mathrm{ph}}\right]$, given by Eqn. (7), we can in fact use Eqn. (13) to find $\zeta(r)$ for all $r$.

\section{Discussion}

For late times when the photosphere becomes negligibly small $\left(r_{\mathrm{ph}} \longrightarrow 0\right)$, the mid region disappears and Eqn. (7) becomes meaningless. We cannot use the reduced quantities $f(z)$ and $s(r)$ in this case. Instead the counterpart to Eqns. (8) and (10) is:

$$
\frac{F(z)}{2 \pi}=\int_{|z|}^{\infty} S(r)[1-\zeta(r)] r d r
$$


which implies that $F(z)$ is symmetric about $z=0$ : i.e., the line profile is symmetric about the rest wavelength. From Eqn. (14) we derive:

$$
S(r)[1-\zeta(r)]=-\frac{1}{2 \pi} \frac{1}{z} \frac{d F}{d z}=-\frac{1}{2 \pi}\left(\frac{\lambda_{0}}{c t}\right)^{2} \frac{1}{\Delta \lambda} \frac{d F}{d \Delta \lambda}
$$

where $r=|z|=c t|\Delta \lambda|$. Since the profile is symmetric one obtains the same information using either $z \leq 0$ or $z \geq 0$. Thus without a photosphere it is not possible to separate $S$ and $\zeta$, however the product $S\{1-\zeta\}$ can be determined for all radii.

To demonstrate that the above equations really do allow for a clean inversion, we have generated line profiles under the given assumptions and using $S(r)$ and $\tau(r)$ given by power laws of various exponents. Figure 2 shows that the power law behavior can be recovered by applying the inversion formulae to the line shape. Although here we have assumed that the functions are monotonically decreasing with $r$, this is not a necessary condition for our derivations and the formulae apply also for non-monotonic distributions.

Because the inversions in Figure 2 were applied to pristine model lines, the results show very little noise (the small amount is due to numerical error), however in application to real data, the quality of the inversion will of course depend upon the signal to noise and spectral resolution of the data. Because derivatives are especially sensitive to a high frequency noise component, smoothing of the spectrum or some other stabilization technique may need to be applied, which typically amounts to assuming a priori some level of smoothness of the functions $\tau(r)$ and $S(r)$ (c.f. Craig \& Brown 1986).

\section{Conclusion}

Eqns. (7), (9), and (13), taken together constitute a complete analytic inversion of supernova lines in the Sobolev approximation. Given the present assumptions, some of the more interesting facts are: (1) the steepness of the mid region reflects the size of the optical depth; (2) the absence of a line implies negligible optical depth; (3) a jagged mid region signals a clumpy absorbing region near the photosphere; $(4)$ the emission feature may have no rising humps; (5) emission features cannot be blueshifted or redshifted simply by varying the line source function or optical depth. When applied under the right circumstances, the formulae may provide useful information on the physical conditions in the atmosphere as well as constraints on supernova explosion models.

It is also interesting that this inversion problem possesses a unique solution for both $S(r)$ and $\tau(r)$. A persistent worry in supernova modeling is that very different physical parameters may lead to identical looking synthetic spectra. The analytic solutions above 
demonstrate that, at least in principle, each different choice of $S(r)$ and $\tau(r)$ produces a distinct line profile (although in practice it may be impossible to discern the differences from noisy data). Although for more general models the inversion will not be unique, the success in the present case does give some support that a good fit to a line does indeed imply realistic physical parameters.

We would like to thank Peter Nugent and R. Ignace for helpful comments and suggestions. This work was supported in part by NSF grants AST-9731450, AST-9986965 and NASA grant NAG5-3505.

\section{REFERENCES}

Branch, D. et al. 1983, ApJ, 270, 123

Castor, J. I. 1970, MNRAS, 149, 111

Castor, J. I. \& Nussbaumer, H. 1972, MNRAS, 155, 293

Chugai, N. N. 1988, Soviet Astronomy Letters, 14, 334

Craig, I. \& Brown, J. 1986, Inverse Problems in Astronomy (Bristol: Adam Hilger Ltd)

Deng, J. S., Qiu, Y. L., Hu, J. Y., Hatano, K., \& Branch, D. 2000, ApJ, 540, 452

Duschinger, M., Puls, J., Branch, D., Höflich, P., \& Gabler, A. 1995, A\&A, 297, 802

Fransson, C. \& Chevalier, R. 1989, ApJ, 343, 323

Hamann, W.-R. 1981, A\&A, 93, 353

Ignace, R. \& Hendry, M. A. 2000, ApJ, 537, L131

Jeffery, D. \& Branch, D. 1990, in Supernovae, ed. J. C. Wheeler \& T. Piran (Singapore: World Scientific), 149

Jeffery, D. J. 1993, ApJ, 415, 734

Mazzali, P. A., Lucy, L., \& Butler, K. 1992, A\&A, 258, 399

Mihalas, D. 1978, Stellar Atmospheres (New York: W. H. Freeman)

Millard, J., Branch, D., Baron, E., Hatano, K., Fisher, A., Filippenko, A. V., et al. 1999, ApJ, 527, 746 
Olson, G. 1982, ApJ, 255, 267

Pauldrach, A., Puls, J., \& Kudritzki, R. P. 1986, A\&A, 164, 86

Rybicki, G. B. \& Hummer, D. G. 1978, ApJ, 219, 654

Sobolev, V. V. 1960, Moving Envelopes of Stars (Cambridge, MA: Harvard Univ. Press)

Wang, L. \& Hu, J. 1994, Nature, 369, 380 


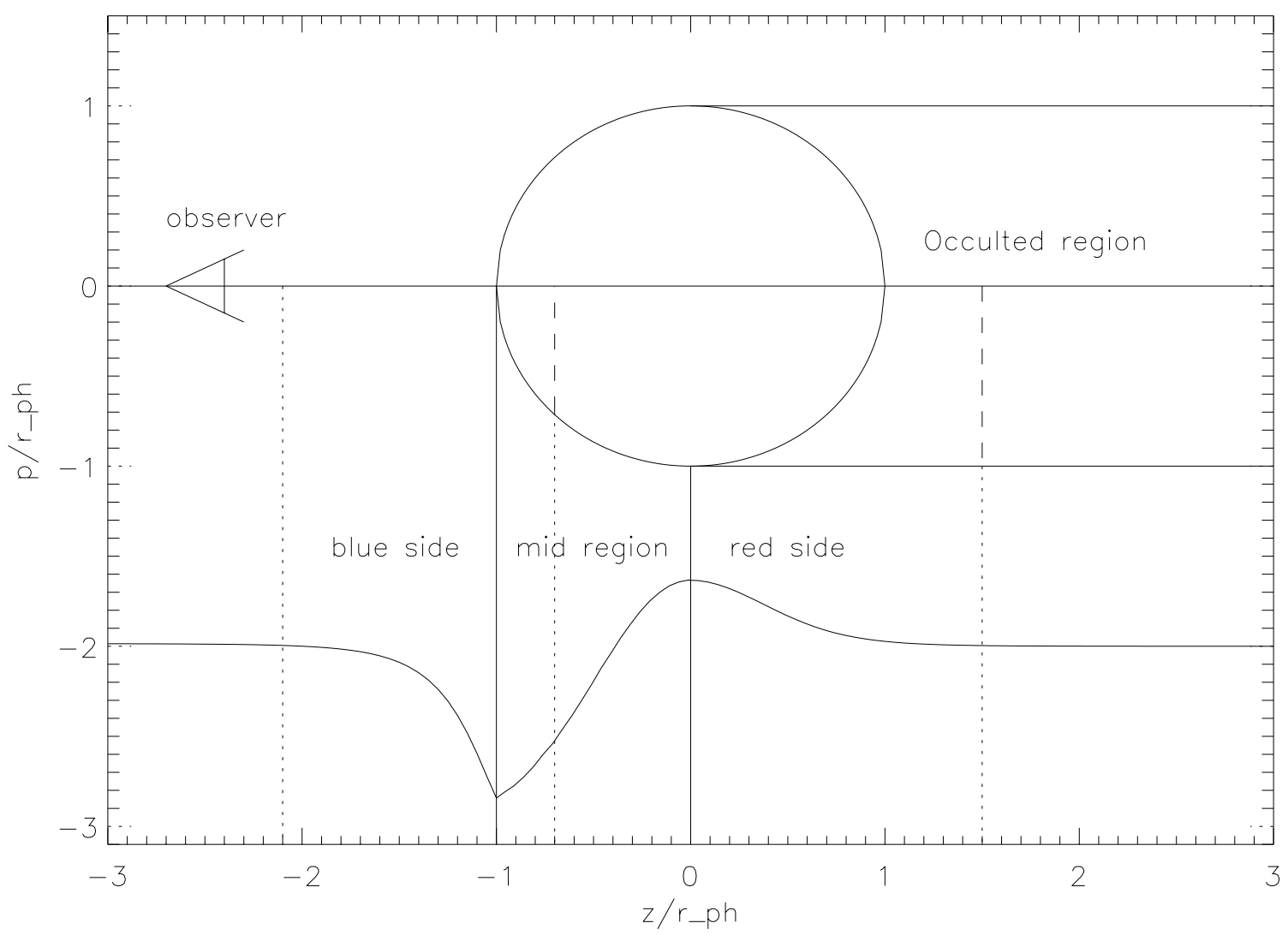

Fig. 1.- Schematic diagram of how line profiles are calculated in the Sobolev approximation. The figure is a cross-sectional view of the supernova with the sphere in the center representing the photosphere. The dotted/dashed lines show the region of integration for three points on the line profile, one each on the red side, the mid region, and the blue side. In each case the integration over that region of the atmosphere produces the flux at the wavelength where the dotted line intersects the line profile. On the mid and red side, the dashed lines represent the region of the atmosphere where light comes directly from the photosphere. 

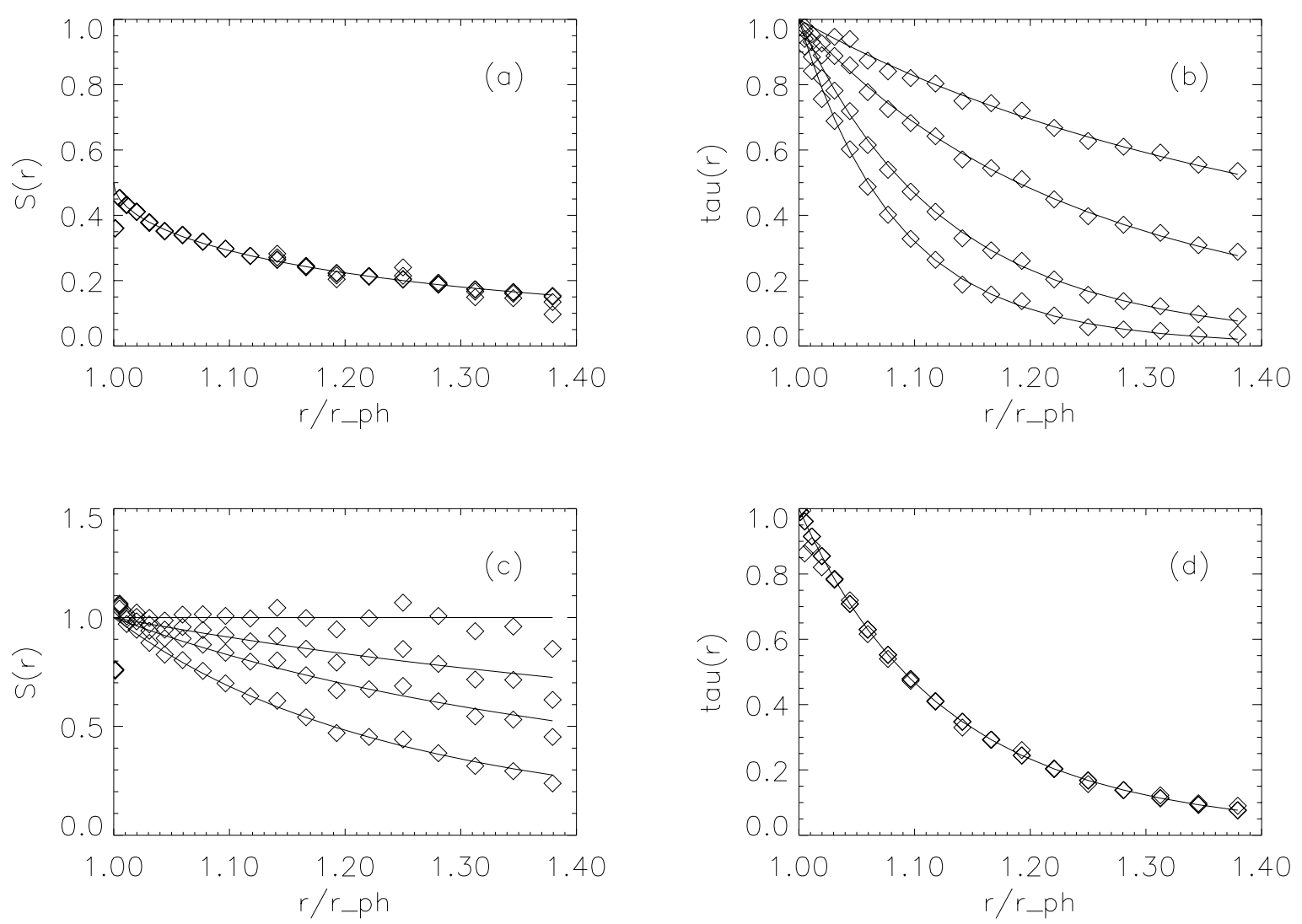

Fig. 2. - Examples of the application of the inversion formulae. Panels (a) and (b) show the source function and optical depth obtained from the inversion of line profiles generated with the same source function $S=W(r)$, but different power indices $(n=2,4,8,12)$ for the optical depth. Panels $(\mathrm{c})$ and $(\mathrm{d})$ show the the source function and optical depth obtained from the inversion of line profiles generated with the same optical depth power law $(n=8)$, but different source functions $\left(S(r) \propto r^{-n}, n=0,1,2,4\right)$. The solid lines are the exact input functions and the diamonds are the functions extracted using the inversion formulae. The small amount of noise in the plots is due to numerical error. 